Case Report

\title{
A Rare Case of 18-year-old female with Primary Sclerosing Cholangitis, Mixed Connective Tissue Disease, Short Stature
}

\author{
Fransisca Yustika Dewi Siahaan ${ }^{1}$, Hery Djagat Purnomo ${ }^{2 *}$, R Y Hellmi ${ }^{3}$, Heri Nugroho ${ }^{4}$ \\ ${ }^{1}$ Department of Internal Medicine, Faculty of Medicine Diponegoro University - Dr. Kariadi Hospital, Semarang, \\ Indonesia \\ ${ }^{2}$ Division of Gastroentero-Hepatology, Department of Internal Medicine, Faculty of Medicine Diponegoro University \\ - Dr. Kariadi Hospital, Semarang, Indonesia \\ ${ }^{3}$ Division of Rheumatology, Department of Internal Medicine, Faculty of Medicine Diponegoro University - Dr. \\ Kariadi Hospital, Semarang, Indonesia \\ ${ }_{4}^{4}$ Division of Endocrinology, Diabetes and Metabolism, Department of Internal Medicine, Faculty of Medicine \\ Diponegoro University - Dr. Kariadi Hospital, Semarang, Indonesia
}

\begin{abstract}
Article Info
History

Received: 16 Dec 2019

Accepted: 16 Mar 2020

Available: 31 Aug 2020

Abstract

Background: Primary sclerosing cholangitis (PSC) is a rare chronic immunemediated liver disease affecting intra and extrahepatic bile ducts, in which inflammation and fibrosis are the main factors that lead to biliary stricture. The pathogenesis of this disease is still unclear. It happens approximately 0.5 to 1.3 cases per 100,000 person-years in Northern Europe. PSC is difficult to diagnose until complications arise. PSC can also be a manifestation of mixed connective tissue disease (MCTD) in the liver. A chronic immune disease that has occurred since childhood may result in delayed development and short stature.

Case Presentation: A 18-year-old female presented with icteric, anemia, short stature, and absence of menstruation. Laboratory and radiological findings suggested obstruction of intra and extrahepatic biliary duct (conjugated bilirubin 11,80 mg/dL), severe anemia, normal sex hormone levels, increased RNP/SM antibodies. Histopathology showed liver cirrhosis suggestive for primary sclerosing cholangitis. The patient then was given biliary stenting, transfusion of packed red cell, immunosuppresive, corticosteroid, ursodeoxycholic acid. Within a year of treatment, there was a significant decrease of direct bilirubin and improvement in symptoms.

Conclusion: Patient with PSC, MCTD, and short stature is a very rare case. This is the first case that has been found in dr. Kariadi Hospital, Semarang. It has high mortality rate and difficult to diagnose and treat. According to multiple guidelines, this patient should have liver transplantation, but in this case, biliary stenting and oral treatments have resulted in an excellent outcome.
\end{abstract}

Keywords: Primary sclerosing cholangitis; MCTD; short stature

Permalink/ DOI: https://doi.org/10.14710/jbtr.v6i2.6299

\section{INTRODUCTION}

Primary sclerosing cholangitis (PSC) is a chronic, progressive, stricturing disease of the biliary tree. Most patients with primary sclerosing cholangitis have no symptoms. This disease is usually detected based on abnormal result of biochemical tests of liver function on routine blood testing.

\footnotetext{
* Corresponding author:

E-mail: herydjagat@yahoo.co.id

(Hery Djagat Purnomo)
}

Patients may remain asymptomatic for many years despite the presence of the advanced disease. When symptoms develop, it will result in obstruction to bile flow, including jaundice, itching, right upper quadrant abdominal pain, fever, and chills. Symptoms may also include weight loss and fatigue. The development of symptoms usually suggests the presence of advanced diseases including liver cirrhosis, portal hypertension, and end-stage liver disease. (1) PSC is strongly associated with inflammatory bowel disease (IBD)(2). It 
appears to be much less common in Southeast Asia. It is most common in Northern European countries and North America, where the reported incidence and prevalence range from approximately 0.5 to 1.3 cases per 100,000 person-years and 3.85 to 16.2 cases per 100,000 person-years, respectively. (3) Most patients with primary sclerosing cholangitis are men $(75 \%)$ with an average age of approximately 40 years at diagnosis. Connective tissue diseases (CTDs) are generally referred to as a group of systemic disorders with abnormalities in the tissues containing collagen and elastin. Usually, CTDs are characterized by overactivity of the immune system from unknown causes resulting in the production of autoantibodies.(4) Further, the liver is frequently involved in connective tissue diseases (CTD), most commonly in the form of liver function test biochemical changes with predominant cholestatic or hepatocellular patterns.(5) The purpose of this report is to present a rare case of primary sclerosing cholangitis that overlap with mixed connective tissue disease (MCTD) and delayed development that has occurred since childhood.

\section{CASE REPORT}

A 18-year-old female presented with yellow discoloration of eyes and body that she had had for the past 4 years. The discoloration started from the eyes then developed throughout her whole body. She also complained of generalized pruritus, weakness, and right upper quadrant discomfort. The abdominal discomfort was intermittent and not referred to other areas. She also had dark urine and acholic stools. The patient also did not have her period and there was no secondary sexual characteristic development. There were no fever, diarrhea, cough, night sweats, changes in mental status, signs of any encephalopathy. She had a history of anemia with repeated blood transfusions.

Physical examination revealed stable hemodynamic, height $142 \mathrm{~cm}$ (short stature), body mass index 13.8 (underweight), icteric sclera and body with scratch mark throughout the body, breast and pubic hair were not developed, liver was not palpable with liver span $7 \mathrm{~cm}$ and splenomegaly (Schuffner 2). There were no ascites, spider naevi, palmar erythema, and white nail. On admission, the laboratory data showed anemia (hemoglobin $7.6 \mathrm{mmol} / \mathrm{L}$, MCV 28,8 fl, MCH $28.8 \mathrm{pg}$, FT4 18,35 pmol/L, TSHS 2,39 IU/mL , aspartate aminotransferase $34 \mathrm{IU} / \mathrm{l}$, alanine aminotransferase 18 IU/l, alkaline phosphatase 796 IU/l, $\gamma$-glutamyl transpeptidase $242 \mathrm{IU} / \mathrm{l}$, total bilirubin $16,1 \mathrm{mg} / \mathrm{dL}$, and direct bilirubin 14,3 mg/dL. The viral hepatitis B and C marker was negative. ANA and Anti dsDNA tests were negative. Increased levels of DFS70 and RNP/Sm antibodies from the ANA profile can be concluded as MCTD. The hormone levels of FSH, prolactin, and estradiol were normal. USG showed hypoplasia of the uterus $(3.27 \times 2.27 \times 1.17 \mathrm{~cm})$. MRCP showed a sclerosing cholangitis appearance. The patient had ERCP and 10 Fr $7 \mathrm{~cm}$ biliary stenting.

Liver biopsy showed hepatic cirrhosis with cholestasis that was suggestive for primary sclerosing cholangitis. Treatment of cyclosporine (25mg/day), methylprednisolone $(32,5 \mathrm{mg} /$ day) for four days and then tapering off to $4 \mathrm{mg} / \mathrm{day}$, and ursodeoxycholic acid (UDCA) $250 \mathrm{mg} / 12$ hour, packed red cell transfusion was given. A month later, the patient's symptoms and laboratory results improved. Seven months after admission, the patient had melena and severe anemia with hemoglobin 6,7 gr\%, MCV $27 \mathrm{fl}, \mathrm{MCH} 27 \mathrm{Pg}$, reticulocyte $4,79 \%$. She was then given a packed red cell transfusion and Esophagogastroduodenoscopy was performed with Grade II esophageal varices as the result.

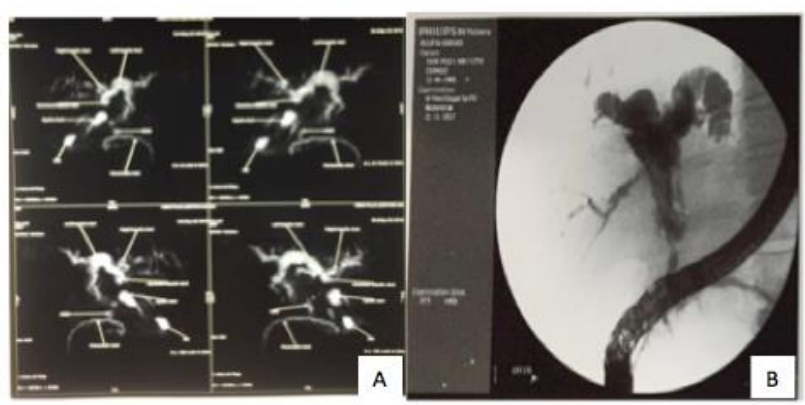

Figure 1. The cholestatic pattern in primary sclerosing cholangitis (A); Biliary stenting in the bile duct (B)
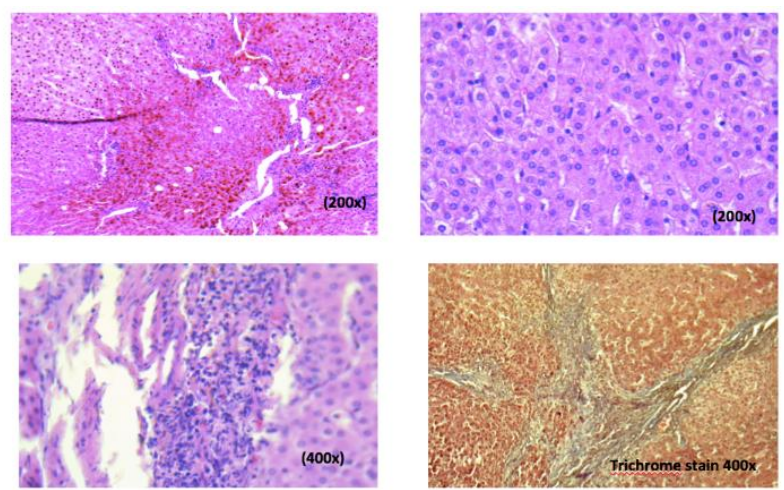

Figure 2. 1-2 Hepatocyte with the oval nucleus, smooth chromatin, trabecular pattern in pseudolobulus, half of the cytoplasm contains bile pigment, and inflammatory cells PMN, lymphocyte. No signs of malignancy and from trichrome stain: Fibrosis (F4).

Once a month the patient was routinely checked and continued her oral medication of cyclosporine (25mg/day), methylprednisolone $4 \mathrm{mg} /$ day, and ursodeoxycholic acid (UDCA) $250 \mathrm{mg} / 12$ hour throughout the year. Her symptoms and laboratory result gradually improved. Approximately a year after the patient had biliary stenting and oral medicine treatment, the yellow discoloration of the body decreased and her direct bilirubin level decreased to $2.21 \mathrm{mg} / \mathrm{dl}$ with a total bilirubin $3.26 \mathrm{mg} / \mathrm{dl}$ and severe anemia became moderate anemia (hemoglobin $8 \mathrm{~g} \%$ ).

\section{DISCUSSION}

We present a patient with primary sclerosing cholangitis (PSC) that has occurred since childhood, a relatively infrequent but increasingly diagnosed disease. PSC is characterized by chronic cholestasis, associated with chronic inflammation of the biliary epithelium, resulting in multi-focal bile duct strictures that can affect the entire biliary tree. Chronic inflammation leads to fibrosis involving the hepatic parenchyma and biliary tree, which can lead to cirrhosis and malignancy. The etiology of PSC is not fully understood. ${ }^{(6)}$ PSC is strongly associated with inflammatory bowel disease. Like many autoimmune diseases, PSC is associated with a range of 
other autoimmune conditions such as connective tissue disease. Clinical manifestations are broad, and the spectrum encompasses asymptomatic cholestasis, icteric cholangitis with pruritus, cirrhosis, and cholangiocarcinoma.. On clinical exam, patients may be unremarkable or present with jaundice and pruritus that can range from mild to disabling, resulting in severe excoriations and a decreased quality of life. The diagnosis of PSC is based on elevated serum markers of cholestasis (AP, $\gamma \mathrm{GT}$, Bilirubin) and imaging findings magnetic resonance cholangiopancreatography (MRCP), endoscopic retrograde cholangiopancreatography (ERCP) demonstrating characteristic changes of bile duct with multifocal strictures and segmental dilatations when all other possible cholestatic disorders and secondary causes are excluded. On biopsy, PSC presents with fibrous obliteration of small bile ducts, with concentric replacement by connective tissue in an "onion skin" pattern. (7) The ppatient repeatedly had anemia and received packed red cell transfusion and Ursodeoxycholic acid (UDCA) for cholestasis as a hydrophilic bile acid, and it has been the most extensively studied putative pharmacological treatment for PSC. ${ }^{(8)}$ Immunosuppressive agent and corticosteroid were given to this patient because of the autoimmune etiology of the disease. Biliary stenting was performed in this patient to help the stream of the bile. ${ }^{(9)}$

Even though studies show that UDCA therapy improve biochemical parameters but the overall beneficial effects for patients with PSC, in particular, survival benefit, are still uncertain. Immunosuppression in PSC is controversial and the majority of centers and publications do not recommend a routine administration of corticosteroids and other immunosuppressants. ${ }^{(10)}$ In this patient, it was objectively proved that UDCA, immunosuppressant, corticosteroid combined with biliary stenting improved symptoms and laboratory results with marked decreased of direct bilirubin and sufficient hemoglobin levels. Her cholestatic pattern can be measured objectively through her laboratory examination. The first direct bilirubin level was 14,3 $\mathrm{mg} / \mathrm{dL}$ before biliary stenting and oral treatment and approximately a year after the treatment, her direct bilirubin level markedly decreased to nearly normal level and her hemoglobin level was increased to nearly normal level. Treatment modality for PSC is still challenging with liver transplantation as the definitive treatment, where the survival rate may increase to 90\%. ${ }^{(11)}$ However, when liver transplantation is not an option, we can consider giving biliary stenting, immunosuppressant, corticosteroid, UDCA as supporting therapy. Even though the mean duration of the stent patency is about 12 months and needs close and regular monitoring and prolonged corticosteroid therapy may give serious side effects, they are still considered an effective treatment modality for this patient that does not have resources for liver transplantation. ${ }^{(12)}$

Her quality of life was improved but the patient still had no menstruation and her secondary sexual sign did not develop. Fibrotic autoimmune diseases are characterized by an inflammatory process, which induces and sustains robust fibrosis. Among the various fibrogenic factors in autoimmune diseases, interleukin-1
(IL-1), IL-6, and TGF- $\beta$ have a relevant role. ${ }^{(13)}$ Short stature may be either a variant of normal growth or caused by a disease. Almost any serious disease can cause growth failure. The abnormalities of growth and maturation that occur in children with acute or chronic illnesses may result from the primary disease process because of increased energy needs or nutritional deprivation (eg, decreased intake or malabsorption). The growth failure is closely related to the inflammatory disease process (mediated by proinflammatory cytokines), as well as decreased food intake, malabsorption, and/or high-dose glucocorticoids if used for treatment. ${ }^{(14)}$ Further examination should be done to establish the cause of her short stature and undeveloped secondary sex characteristics to give the proper treatment.

\section{CONCLUSION}

PSC is a rare cholestatic disease with unclear etiology. Liver involvement in MCTD patients is also rare. Chronic diseases can also decrease body height through their effects on overall health. Even though there is no definitive proof that stenting and oral treatment give improvement in PSC patients, but in this case, it is objectively proved that they diminished cholestatic pattern of this patient. However, liver transplantation is still a definitive therapy for PSC patient. Further studies are needed for better understanding of the disease and for development of an optimal therapeutic strategy for patients with PSC.

\section{ACKNO.WLEDGEMENT}

We would like to thank all the doctors that involved in diagnosing and treating this patient. The authors declare that they have no conflict of interest.

\section{REFERENCES}

1. Chapman R, Fevery J, Kalloo A, Nagorney DM, Boberg KM, Shneider B, Gores GJ. Diagnosis and management of primary sclerosing cholangitis. Hepatology. 2010 Feb;51(2):660-78.

2. Fousekis FS, Theopistos VI, Mitselos IV, Skamnelos A, Kavvadias A, Katsanos KH, Christodoulou DK. Specific Features of Patients With Inflammatory Bowel Disease and Primary Sclerosing Cholangitis. Journal of clinical medicine research. $2019 \mathrm{Feb} ; 11(2): 81$.

3. Tabibian JH, Ali AH, Lindor KD. Primary sclerosing cholangitis, Part 1: Epidemiology, etiopathogenesis, clinical features, and treatment. Gastroenterology \& hepatology. 2018 May;14(5):293.

4. Grutters JC, Lammers JW. Connective Tissue Diseases. InClinical Respiratory Medicine 2012 Jan 1 (pp. 653-666). WB Saunders.

5. De Santis M, Crotti C, Selmi C. Liver abnormalities in connective tissue diseases. Best Practice \& Research Clinical Gastroenterology. 2013 Aug 1;27(4):543-51.

6. Eaton JE, Talwalkar JA, Lazaridis KN, Gores GJ, Lindor KD. Pathogenesis of primary sclerosing cholangitis and advances in diagnosis and management. Gastroenterology. 2013 Sep 1;145(3):521-36. 
7. Sirpal S, Chandok N. Primary sclerosing cholangitis: diagnostic and management challenges. Clinical and experimental gastroenterology. 2017;10:265.

8. Virani S, Akers A, Stephenson K, Smith S, Kennedy L, Alpini G, Francis H. Comprehensive Review of Molecular Mechanisms during Cholestatic Liver Injury and Cholangiocarcinoma. Journal of liver. 2018;7(3).

9. Abu-Wasel B, Keough V, Renfrew PD, Molinari M. Biliary stent therapy for dominant strictures in patients affected by primary sclerosing cholangitis. Pathobiology. 2013;80(4):182-93.

10. Goode EC, Rushbrook SM. A review of the medical treatment of primary sclerosing cholangitis in the $21 \mathrm{st}$ century. Therapeutic advances in chronic disease. 2016 Jan;7(1):68-85.
11. Chapman MH, Thorburn D, Hirschfield GM, Webster GG, Rushbrook SM, Alexander G, Collier J, Dyson JK, Jones DE, Patanwala I, Thain C. British Society of Gastroenterology and UK-PSC guidelines for the diagnosis and management of primary sclerosing cholangitis. Gut. 2019 Jun 1:gutjnl-2018.

12. Fung BM, Tabibian JH. Biliary endoscopy in the management of primary sclerosing cholangitis and its complications. Liver research. 2019 Apr 3.

13. Chentoufi AA, Serov YA, Alazmi M, Baba K. Immune components of liver damage associated with connective tissue diseases. Journal of clinical and translational hepatology. 2014 Mar;2(1):37.

14. Rogol A D. Causes of short stature. In: Snyder PJ, Geffner ME, editors. UpToDate [Internet]. Waltham (MA): UpToDate Inc; 2019 [cited 2019 Jul 14]. 